\title{
Staphylococcus aureus: Screening for Nasal Carriers in a Community Setting with Special Reference to MRSA
}

\author{
Yukti Sharma, ${ }^{1}$ Sanjay Jain, ${ }^{1}$ Harshvardhan Singh, ${ }^{2}$ and Vasudha Govil ${ }^{1}$ \\ ${ }^{1}$ Department of Microbiology, Hindu Rao Hospital, Delhi 110007, India \\ ${ }^{2}$ Department of Biochemistry, Hindu Rao Hospital, Delhi 110007, India \\ Correspondence should be addressed to Yukti Sharma; dryukti2006@yahoo.com
}

Received 21 January 2014; Accepted 4 June 2014; Published 25 June 2014

Academic Editor: Muzaffar Hussain

Copyright (c) 2014 Yukti Sharma et al. This is an open access article distributed under the Creative Commons Attribution License, which permits unrestricted use, distribution, and reproduction in any medium, provided the original work is properly cited.

\begin{abstract}
Introduction. Emergence of MRSA infections among previously healthy persons in community settings (without exposure to health care facilities) has been noted recently. MRSA infections are now classified as health care-associated MRSA (HA-MRSA) and community-associated MRSA (CA-MRSA) infections. Its colonization is an important risk factor for subsequent MRSA infection. Aims and Objectives. The aim was to screen patients and health care workers for staphylococcal carriage, identify risk factors for MRSA colonization, and determine the sensitivity pattern. Materials and Methods. A total of 200 subjects were screened for nasal carriage after obtaining verbal consent. These were both healthy subjects attending various outpatient departments and health care workers. Specimens were collected from the anterior nares using premoistened sterile cotton swabs and inoculated onto blood agar and mannitol salt agar and incubated at $37^{\circ} \mathrm{C}$ for $24-48 \mathrm{~h}$. Results. Staphylococcus aureus colonisation was found to be $12 \%(n=24)$. MRSA was identified in 5\% $(n=10)$ which represents $41.66 \%$ of SA. A total of 10 strains of MRSA were isolated from 200 subjects, giving an overall positivity rate of 5\%. Discussion. Staphylococcal colonization was found to be $12 \%$ (MRSA 5\%). Fluoroquinolone resistance was remarkable whereas all strains were sensitive to vancomycin, teicoplanin, linezolid, quinupristin-dalfopristin.
\end{abstract}

\section{Introduction}

The emergence of MRSA infections among previously healthy persons in community settings (without exposure to health care facilities) has been noted recently $[1,2]$. Therefore, MRSA infections are now classified as health care-associated MRSA (HA-MRSA) infections and community-associated MRSA (CA-MRSA) infections [3]. Apparently acquired in the community, these infections are termed as community-associated MRSA infections (CA-MRSA) [4, 5]. CA-MRSA strains and health care-associated (HA) MRSA strains differ in terms of epidemiology, microbiology, and clinical manifestations [5]. Colonization rates of MRSA in the community have been reported to range from 0 to 9.2 percent [6]. The nose and open skin areas (i.e., wounds and device exit sites) are considered the most important sites for colonization [710]. Nasal carriage of MRSA is an important risk factor for subsequent MRSA infection and transmission of this pathogen [11]. Active surveillance for patients colonized with methicillin resistant Staphylococcus aureus (MRSA) is recommended to prevent MRSA infections in health care settings $[7,12]$. Although several studies have reported the prevalence of MRSA nasal carriage in patients in health care settings $[13,14]$, this subject has been little investigated in healthy individuals in the broader community [15]. Infection control teams in local Delhi hospitals expressed concern that an increasing number of patients were being admitted to hospital already colonized with MRSA. It was suggested that there might be a high prevalence of MRSA in the local community and so we undertook a prevalence survey of MRSA colonization in the general population in Delhi. The objectives of the present study were to screen patients and health care workers for MRSA carriage, to identify risk factors for MRSA colonization, and to determine their resistance pattern. CA-MRSA is responsible for around 30 percent of S. aureus infections in hospitals of USA [16].

There is a growing urgency to study the colonization behaviour of CA-MRSA. 


\section{Materials and Methods}

A total of 200 subjects (170 outpatients and 30 health care workers) were screened for MRSA after obtaining verbal consent from subjects. Using premoistened sterile cotton swabs, specimens were collected from the anterior nares of patients and health care workers.

The specimens were inoculated onto sheep blood agar and mannitol salt agar and incubated at $37^{\circ} \mathrm{C}$ in ambient air for 24-48 h. Colonies suggestive of S. aureus (white or cream haemolytic or nonhaemolytic colonies on oxacillin blood agar and yellow colonies on mannitol salt agar) were identified using Gram stain, catalase, and slide and tube coagulase tests. The isolates were confirmed as MRSA by disc diffusion (1 $\mu \mathrm{g}$ oxacillin).

2.1. Drug Susceptibility Tests. The antimicrobial susceptibility of $S$. aureus isolates was assessed by disk-diffusion tests, according to the Clinical Laboratory Standards Institute guidelines $[17,18]$. The following antimicrobial discs were used: oxacillin $(1 \mu \mathrm{g})$, ciprofloxacin $(5 \mu \mathrm{g})$, gentamicin $(10 \mu \mathrm{g})$, amikacin $(30 \mu \mathrm{g})$, linezolid $(30 \mu \mathrm{g})$, quinpristin-dalfopristin, cefoperazone $(30 \mu \mathrm{g})$, and vancomycin $(30 \mu \mathrm{g})$. All the discs were obtained from Hi-media (Hi-media, Mumbai, India). $S$. aureus ATCC 25923 was used as the internal control in each run of the test.

2.2. Data Collection. A standardized questionnaire was used to collect information on the risk factors for CA-MRSA colonization. The data collected were age, sex, educational degree, and marital status and whether the subject was living in a hostel or not, the number of household members, the presence of any household member who was a health care worker (HCW), the presence of any household member who was less than 7 years old, the presence of any household member who was bedridden, the presence of chronic diseases, smoking habits, hospitalizations within the previous year, a history of caring for inpatients within the past year, outpatient clinic visits within the past year, the use of antibiotics within the previous year, a history of skin and soft-tissue infection within the previous year, whether the subject takes a shower every day, and economic status.

2.3. Statistics. Statistical analysis of the risk factor association with SA and MRSA colonization was calculated using the Chi-square test (Epi info software).

\section{Results}

During the 2-month study period, there were 200 people enrolled. Staphylococcus aureus colonisation was found in $12 \%$ (SA $=24, n=200)$ of participants. Methicillin resistant SA was identified in 5\% (10/200). A total of 10 of these 24 people carried MRSA (41.66\% of SA) and 14 had MSSA (58.33\% of SA). The prevalence of SA and MRSA was analyzed against the surveyed factors (Table 1). One group demonstrated statistically significant correlation with SA: participants visiting OPD in the past year $(P=0.002)$. The
TABLE 1: Calculated correlation between SA nasal and MRSA nasal colonization and various other surveyed factors.

\begin{tabular}{|c|c|c|c|c|}
\hline Parameter & MRSA & MSSA & $\begin{array}{l}P \text { value } \\
\text { (MRSA) }\end{array}$ & $\begin{array}{l}P \text { value } \\
(\text { SA) }\end{array}$ \\
\hline \multicolumn{5}{|l|}{ Sex } \\
\hline Male & 3 & 7 & 0.442 & 0.9736 \\
\hline Female & 7 & 7 & 0.4063 & 0.89 \\
\hline \multicolumn{5}{|l|}{ Education } \\
\hline Elementary school & 8 & 9 & 0.141 & 0.171 \\
\hline Junior high school & 2 & 5 & 0.141 & 0.171 \\
\hline \multicolumn{5}{|l|}{ Senior high school } \\
\hline \multicolumn{5}{|l|}{ Marital status } \\
\hline Married & & 6 & 15 & 0.466 \\
\hline Unmarried & & 4 & 9 & 0.3911 \\
\hline \multicolumn{5}{|l|}{$\begin{array}{l}\text { Presence of members } \\
<7 \text { years of age }\end{array}$} \\
\hline Yes $n=84$ & 3 & 9 & 0.442 & 0.427 \\
\hline \multicolumn{5}{|l|}{$\begin{array}{l}\text { Presence of } \\
\text { bedridden members }\end{array}$} \\
\hline Yes $n=16$ & 1 & 3 & 0.815 & 0.416 \\
\hline \multicolumn{5}{|l|}{ Chronically ill } \\
\hline Yes $n=33$ & 3 & 2 & 0.25 & 0.567 \\
\hline \multicolumn{5}{|l|}{ Smoking habits } \\
\hline Yes & 2 & 5 & 0.694 & 0.142 \\
\hline \multicolumn{5}{|l|}{ Shower everyday } \\
\hline Yes $n=160$ & 8 & 12 & 1 & 0.6831 \\
\hline $\begin{array}{l}\text { Members who are } \\
\text { HCW }\end{array}$ & & & & \\
\hline
\end{tabular}

\begin{tabular}{lcccc} 
Yes $n=30$ & 2 & 2 & 0.657 & 0.819 \\
\hline \multicolumn{3}{c}{ Factors related to exposure in the previous year } \\
$\begin{array}{l}\text { Hospitalization } \\
\quad \text { Yes } n=53\end{array}$ \\
$\begin{array}{l}\text { Cared for in-patients } \\
\quad \text { Yes } n=53\end{array}$ \\
$\begin{array}{l}\text { Used antibiotic } \\
\quad \text { Yes } n=104\end{array}$
\end{tabular}

prevalence of SA in healthcare workers was $13.33 \%(4 / 30)$. MRSA and MSSA were observed amongst 2 members each (MRSA 6.66\%, MSSA 6.66\%).

3.1. MRSA-Positive Individuals. Seven of the ten MRSA isolates were from females (Table 2 ). The mean age of those affected was 28.05 years. Six of the ten $(6 / 10=60 \%)$ carriers reported previous contact with health-care facilities. Subjects who were MRSA-positive were compared with all other subjects and with subjects who were colonized with methicillin sensitive $S$. aureus with respect to previous hospital 
TABLE 2: Antibiotic resistance towards SA and MRSA.

\begin{tabular}{lcc}
\hline Antibiotics & SA resistance & MRSA resistance \\
\hline Oxacillin & 5 & 5 \\
Augmentin & 37.5 & 30 \\
Gentamicin & 25 & 60 \\
Amikacin & 16.66 & 40 \\
Cefuroxime & 8.33 & 20 \\
Ciprofloxacin & 54.16 & 70 \\
Vancomycin & 0 & 0 \\
linezolid & 0 & 0 \\
Quinupristin-dalfopristin & 0 & 0 \\
Teicoplanin & 0 & 0 \\
\hline
\end{tabular}

admission, recent antibiotic use, any contact with a health facility, and all other variables. No significant difference was found between MRSA-positive subjects and others using the chi-square test and Fisher's exact method.

Antibiotic susceptibility: Ciprofloxacin and Gentamicin showed $70 \%$ and $60 \%$ resistance towards MRSA, respectively. Vancomycin, Linezolid, and Quinupristin-dalfopristin were found to be $100 \%$ sensitive in the present study. Only one MRSA strain was found to be fully sensitive to all the drugs. No strain was found to be absolutely resistant to all drugs. (Table 2, Figure 1).

\section{Discussion}

Community-associated methicillin-resistant Staphylococcus aureus (MRSA) is emerging as a leading cause of skin and soft-tissue infections in many parts of the world [1]. Recently, many cases of MRSA infection have been reported in healthy community individuals with no traditional risk factors for MRSA infection [2].

$S$. aureus nasal carriage rate in the present study was found to be $12 \%(24 / 200)$. Usually quoted figures for $S$. aureus nasal carriage is $20-40 \%$ [19]. A study on healthy contacts of outpatient paediatric cases from an urban community at Delhi showed an alarming colonization rate of 5.3 percent [20]. A study from Japan reported $36 \%$ of S. aureus in nares of Japanese adults and $32.4 \%$ in nasal cavity of adults in USA, which is slightly higher in comparison to results of this study [21]. None of the carriers had any underlying conditions like diabetes or hypertension.

MRSA nasal carriage prevalence was found to be $5 \%$ $(10 / 200)$ in the present study. Other community-based studies have also found low MRSA nasal carriage prevalence. The overall MRSA carriage rate of $8.5 \%$ was observed from South India [22]. Munckhof et al. found a prevalence of only $0.7 \%$ among 699 patients in Queensland, Australia [23]. A higher propensity was observed amongst MRSA strains reported in USA (24.15\%) [24]. Taiwan and Zaria, Nigeria, reported a prevalence of $13.6 \%$ and $14.85 \%$, respectively, from anterior nares of healthy population, adults and school pupils [25].

Seven of the ten MRSA isolates were from females, although it was not statistically significant (Table 2). A study from South India reported males to be the major carriers

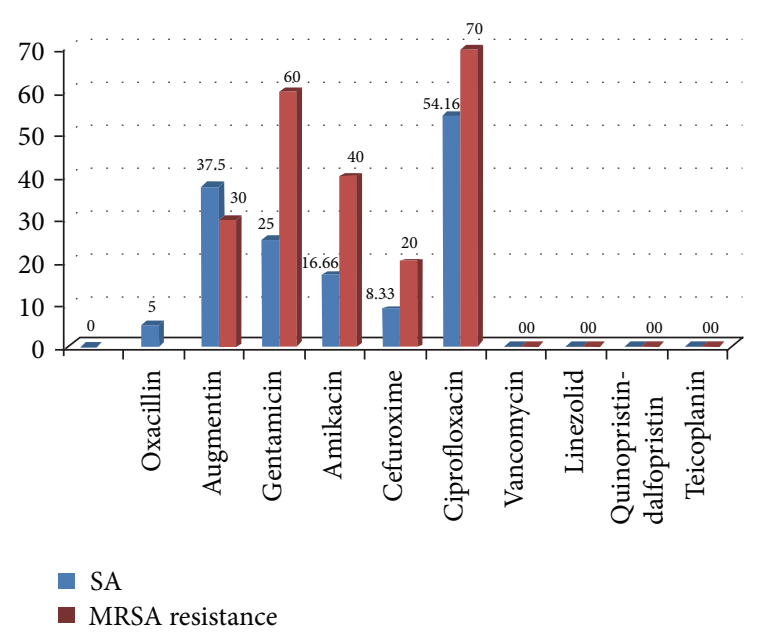

Figure 1: Comparative resistance of SA and MRSA.

$(15 / 118,12.4 \%)[22]$. The mean age of those affected was 28.05 years. Six of the ten $(6 / 10=60 \%)$ carriers reported previous contact with health-care facilities.

Statistical analysis of the risk factor association with SA and MRSA colonization was calculated using the Chi-square test (Epi info software). Risk factors for MRSA colonization were identified by comparing people with MRSA colonization to those with MSSA colonization and people with MRSA colonization to those without carriage of $S$. aureus at the same time. This allowed us to avoid problems associated with multiple intergroup comparisons. The analysis indicated that visiting health-care facility in the past year was an independent risk factor for SA colonization compared to those without $S$. aureus colonization. Studies in the developed world support these findings and suggest that factors associated with CA-MRSA carriage include prior antibiotic usage, contact with health care facility, poor socioeconomic conditions, and overcrowding [22, 23].

During the study we also found that the prevalence of SA in healthcare workers was $13.33 \%(4 / 30)$ which is less as compared to that reported by another study from Delhi $(37.3 \%)$ [26]. Studies elsewhere have reported $6-50 \%$ as carrier rate amongst health care workers, particularly those posted in the burns and intensive care units [22]. In the present study MRSA and MSSA were observed amongst 2 members each (MRSA 6.66\%, MSSA 6.66\%). These results are in consensus with findings by Goyal et al. (MRSA 6.6\% among HCW) [26]. Prevention of MRSA infections merits discussion as once introduced in a hospital, MRSA is very difficult to eradicate [22]. Within hospital, MRSA spreads rapidly by hands of medical personnel. Colonised employees of hospital such as asymptomatic nasal and hand carriers acting as reservoirs are important sources for spreading this organism. Multiple, prolonged use of antibiotics and prolonged hospitalization are other important factors which make hospital an ideal place of transmission and perpetuation of MRSA.

The susceptibility test results showed ciprofloxacin to be the least effective agent with sensitivity being $54.16 \%$. The present study shows ciprofloxacin resistance towards SA to 
be $60 \%$ which is in sharp contrast to the results observed by Chatterjee et al. with $2.4 \%$ [6].

Antibiotic sensitivity pattern of the MRSA isolates (Table 2) shows $70 \%$ resistance to ciprofloxacin $(5 \mu \mathrm{g})$ and $100 \%$ sensitivity to Vancomycin $(30 \mu \mathrm{g})$. These findings are consistent with a study from Delhi [26] where 60\% MRSA were resistant to ciprofloxacin along with $100 \%$ sensitivity towards Vancomycin, but in sharp contrast to the results by Chatterjee et al. [6] with resistance to ciprofloxacin being $12.5 \%$. None of the isolates were multidrug resistant in the present study unlike Goyal et al. who reported 30\% (3/10) resistance to all the antibiotics tested $[26,27]$. It is alarming to note that $60 \%(6 / 10)$ isolates were resistant to ciprofloxacin since it had been proposed as an alternative for treatment of MRSA infection [28]. Increasing number of resistant strains may be the result of selection pressure owing to uncontrolled drug usage in the community.

The survey described here has several limitations. Firstly, active surveillance using only specimens from the anterior nares to screen for MRSA colonization will detect fewer than $75 \%$ of carriers [21]. Nasal cultures may underestimate the real prevalence of $S$. aureus colonization, since it can also be found in other parts of the body as well, such as axilla and pharynx [7]. Specimens other than nasal swabs have been omitted from screening strategies for cost savings, and current recommendations do not emphasize their importance $[19,20]$.

Secondly, the present study's estimation of MRSA nasal carriers is based on the resistance to cefoxitin disc (30 $\mu \mathrm{g})$ and not on the detection of mecA gene that codes for the production of the altered penicillin binding protein (PBP2a) responsible for classical methicillin resistance. A study from Zaria, Nigeria, reported absence of $m e c A$ gene in MRSA isolates [29]. MRSA without $m e c A$ gene are also being implicated in the cause of some severe infections in communities and hospitals [29]. This emphasizes further study on the potentials of these MRSA isolates.

Lastly, these cases may represent carriage of hospital acquired strains in the community rather than transmission within the community. Further investigation is required to confirm this. This issue must be actively addressed to tackle the public health concern of widespread antibiotic resistance. The prevalence of $S$. aureus nasal carriage varies according to the quality of sampling, culture techniques, and the population studied $[1,6,18]$.

\section{Conclusion}

In conclusion, the present study showed that the rate of $S$. aureus nasal carriage was found to be $12 \%(24 / 200)$ and MRSA nasal carriage prevalence was found to be 5\% (10/200).

This study also identifies that visiting health-care facility in the past year was an independent risk factor for SA colonization. These findings could be helpful in controlling the spread of MRSA in community settings. We believe that MRSA surveillance studies in the community setting should be encouraged to gain a better understanding of clinical and molecular epidemiology of the emerging CA-MRSA isolates.

\section{Disclosure}

Presentation at a meeting: poster presentation at 36th National Conference of Indian Association of Medical Microbiologists, from November 22 to November 25, 2012, LHMC.

\section{Conflict of Interests}

The authors declare that there is no conflict of interests regarding the publication of this paper.

\section{References}

[1] Centers for Disease Control and Prevention, "Four pediatric deaths from community-acquired methicillin-resistant Staphylococcus aureus-Minnesota and North Dakota, 1997-1999," Morbidity and Mortality Weekly Report, vol. 48, pp. 707-710, 1999.

[2] T. S. Naimi, K. H. LeDell, K. Como-Sabetti et al., "Comparison of community- and health care-associated methicillinresistant Staphylococcus aureus infections," The Journal of the American Medical Association, vol. 290, no. 22, pp. 2976-2984, 2003.

[3] J. T. Weber, "Community-associated methicillin-resistant Staphylococcus aureus," Clinical Infectious Diseases, vol. 41, no. 4, pp. S269-S272, 2005.

[4] J. W. Beam and B. Buckley, "Community-acquired methicillinresistant Staphylococcus aureus: prevalence and risk factors," Journal of Athletic Training, vol. 41, no. 3, pp. 337-340, 2006.

[5] B. C. Millar, A. Loughrey, J. S. Elborn, and J. E. Moore, "Proposed definitions of community-associated meticillin-resistant Staphylococcus aureus (CA-MRSA)," Journal of Hospital Infection, vol. 67, no. 2, pp. 109-113, 2007.

[6] S. S. Chatterjee, P. Ray, A. Aggarwal, A. Das, and M. Sharma, "A community-based study on nasal carriage of Staphylococcus aureus," Indian Journal of Medical Research, vol. 130, no. 6, pp. 742-748, 2009.

[7] J. E. Coia, G. J. Duckworth, D. I. Edwards et al., "Guidelines for the control and prevention of meticillin-resistant Staphylococcus aureus (MRSA) in healthcare facilities," Journal of Hospital Infection, vol. 63, supplement 1, pp. S1-S44, 2006.

[8] O. Meurman, M. Routamaa, and R. Peltonen, "Screening for methicillin-resistant Staphylococcus aureus: which anatomical sites to culture?" Journal of Hospital Infection, vol. 61, no. 4, pp. 351-353, 2005.

[9] Provincial Infectious Diseases Advisory Committee, Best Practices for Infection Prevention and Control of Resistant Staphylococcus aureus and Enterococci, Ministry of Health and LongTerm Care, Toronto, Canada, 2007.

[10] A. Scanvic, L. Denic, S. Gaillon, P. Giry, A. Andremont, and J.-. Lucet, "Duration of colonization by methicillin-resistant Staphylococcus aureus after hospital discharge and risk factors for prolonged carriage," Clinical Infectious Diseases, vol. 32, no. 10, pp. 1393-1398, 2001.

[11] C. A. Muto, J. A. Jernigan, B. E. Ostrowsky et al., "SHEA guideline for preventing nosocomial transmission of multidrugresistant strains of Staphylococcus aureus and Enterococcus," Infection Control and Hospital Epidemiology, vol. 24, no. 5, pp. 362-386, 2003. 
[12] S. G. Weber, S. S. Huang, S. Oriola et al., "Legislative mandates for use of active surveillance cultures to screen for methicillinresistant Staphylococcus aureus and vancomycin-resistant enterococci: position statement from the joint SHEA and APIC task force," Infection Control and Hospital Epidemiology, vol. 28, no. 3, pp. 249-260, 2007.

[13] D. K. Warren, R. M. Guth, and C. M. Coopersmith, "Epidemiology of methicillin-resistant Staphylococcus aureus colonization in a surgical intensive care unit," Infection Control and Hospital Epidemiology, vol. 27, pp. 1032-1040, 2006.

[14] P. Muñoz, J. Hortal, M. Giannella et al., "Nasal carriage of $S$. aureus increases the risk of surgical site infection after major heart surgery," Journal of Hospital Infection, vol. 68, no. 1, pp. 25-31, 2008.

[15] M. J. Kuehnert, D. Kruszon-Moran, H. A. Hill et al., "Prevalence of Staphylococcus aureus nasal colonization in the United States, 2001-2002," Journal of Infectious Diseases, vol. 193, no. 2, pp. 172179, 2006.

[16] S. L. Kaplan, K. G. Hulten, B. E. Gonzalez et al., "Threeyear surveillance of community-acquired Staphylococcus aureus infections in children," Clinical Infectious Diseases, vol. 40, no. 12, pp. 1785-1791, 2005.

[17] Clinical and Laboratory Standards Institute, "Performance standards for antimicrobial disk susceptibility test," Approved Standard M2-A9, Clinical and Laboratory Standards Institute, Wayne, Pa, USA, 2006.

[18] Clinical and Laboratory Standards Institute, "Performance standards for antimicrobial susceptibility testing," Approved Standard S17, Clinical and Laboratory Standards Institute, Wayne, Pa, USA, 2007.

[19] J. Kluytmans, A. van Belkum, and H. Verbrugh, "Nasal carriage of Staphylococcus aureus: epidemiology, underlying mechanisms, and associated risks," Clinical Microbiology Reviews, vol. 10, no. 3, pp. 505-520, 1997.

[20] S. Saxena, K. Singh, and V. Talwar, "Methicillin-resistant Staphylococcus aureus prevalence in community in the East Delhi area," Japanese Journal of Infectious Diseases, vol. 56, no. 2, pp. 54-56, 2003.

[21] R. C. Moellering Jr., "Introduction: problems with antimicrobial resistance in gram-positive cocci," Clinical Infectious Diseases, vol. 26, no. 5, pp. 1177-1178, 1998.

[22] S. Mathanraj, S. Sujatha, K. Sivasangeetha, and S. Parija, "Screening for methicillin-resistant Staphylococcus aureus carriers among patients and health care workers of a tertiary care hospital in south India," Indian Journal of Medical Microbiology, vol. 27, no. 1, pp. 62-64, 2009.

[23] W.J. Munckhof, G. R. Nimmo, J. M. Schooneveldt et al., "Nasal carriage of Staphylococcus aureus, including communityassociated methicillin-resistant strains, in Queensland adults," Clinical Microbiology and Infection, vol. 15, no. 2, pp. 149-155, 2009.

[24] A. Currie, L. Davis, E. Odrobina et al., "Sensitivities of nasal and rectal swabs for detection of methicillin-resistant Staphylococcus aureus colonization in an active surveillance program," Journal of Clinical Microbiology, vol. 46, no. 9, pp. 3101-3103, 2008.

[25] A. Onanuga and T. C. Temedie, "Nasal carriage of multidrug resistant Staphylococcus aureus in healthy inhabitants of Amassoma in Niger delta region of Nigeria," African Health Sciences, vol. 11, no. 2, pp. 176-181, 2011.

[26] R. Goyal, S. Das, and M. Mathur, "Colonisation of methicillin resistant Staphylococcus aureus among health care workers in a tertiary care hospital of Delhi," Indian Journal of Medical Sciences, vol. 56, no. 7, pp. 321-324, 2002.

[27] L. M. Deshpande, A. M. Fix, M. A. Pfaller, and R. N. Jones, "Emerging elevated mupirocin resistance rates among staphylococcal isolates in the SENTRY Antimicrobial Surveillance Program (2000): correlations of results from disk diffusion, Etest and reference dilution methods," Diagnostic Microbiology and Infectious Disease, vol. 42, no. 4, pp. 283-290, 2002.

[28] K. A. Prates, A. M. Torres, L. B. Garcia, S. F. Y. Ogatta, C. L. Cardoso, and M. C. B. Tognim, "Nasal carriage of methicillinresistant Staphylococcus aureus in university students," Brazilian Journal of Infectious Diseases, vol. 14, no. 3, pp. 316-318, 2010.

[29] B. O. Olayinka, A. T. Olayinka, A. F. Obajuluwa, J. A. Onaolapo, and P. F. Olurinola, "Absence of $m e c A$ gene in methicillinresistant Staphyloccous aureus isolates," African Journal of Infectious Diseases, vol. 3, no. 2, pp. 49-56, 2009. 

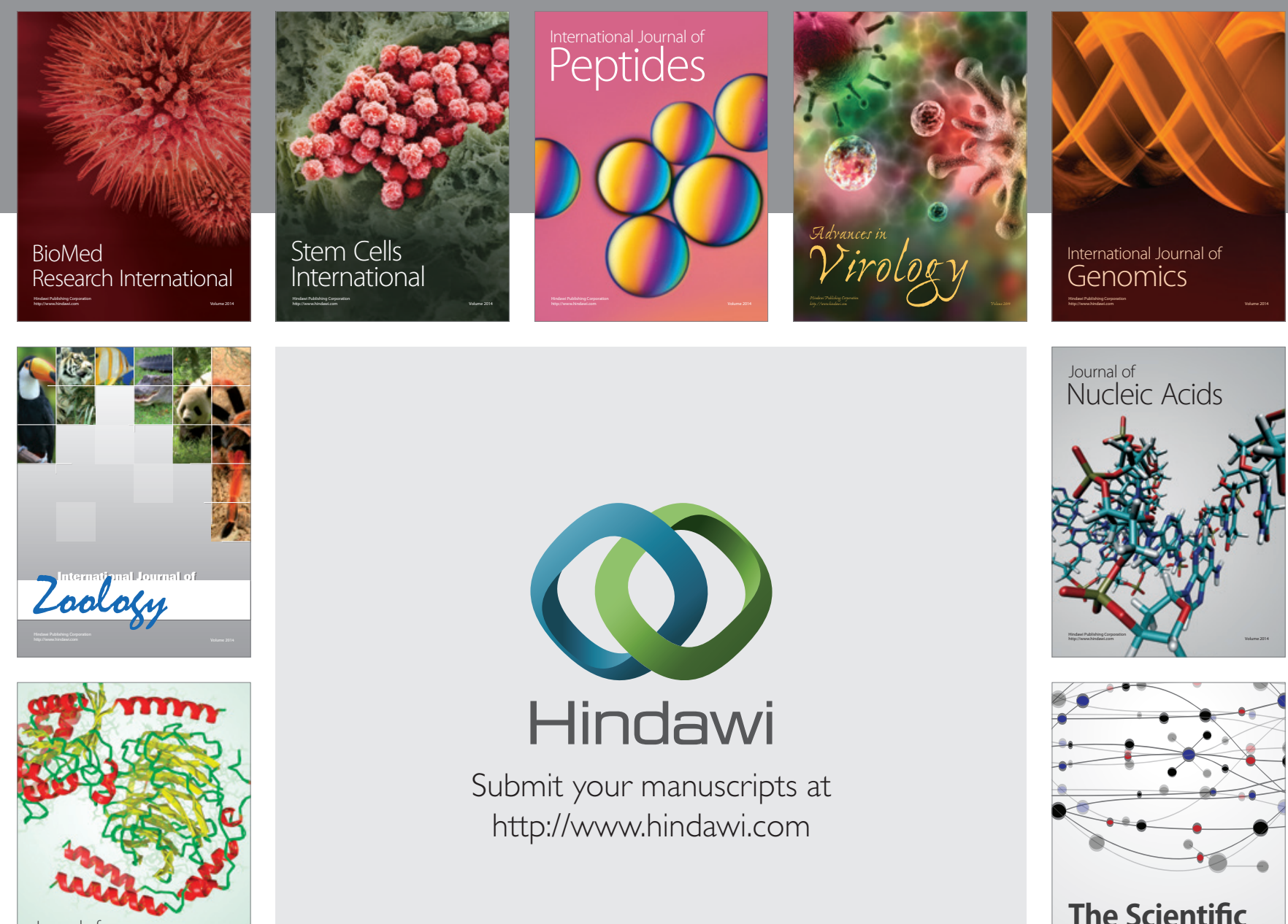

Submit your manuscripts at

http://www.hindawi.com

Journal of
Signal Transduction
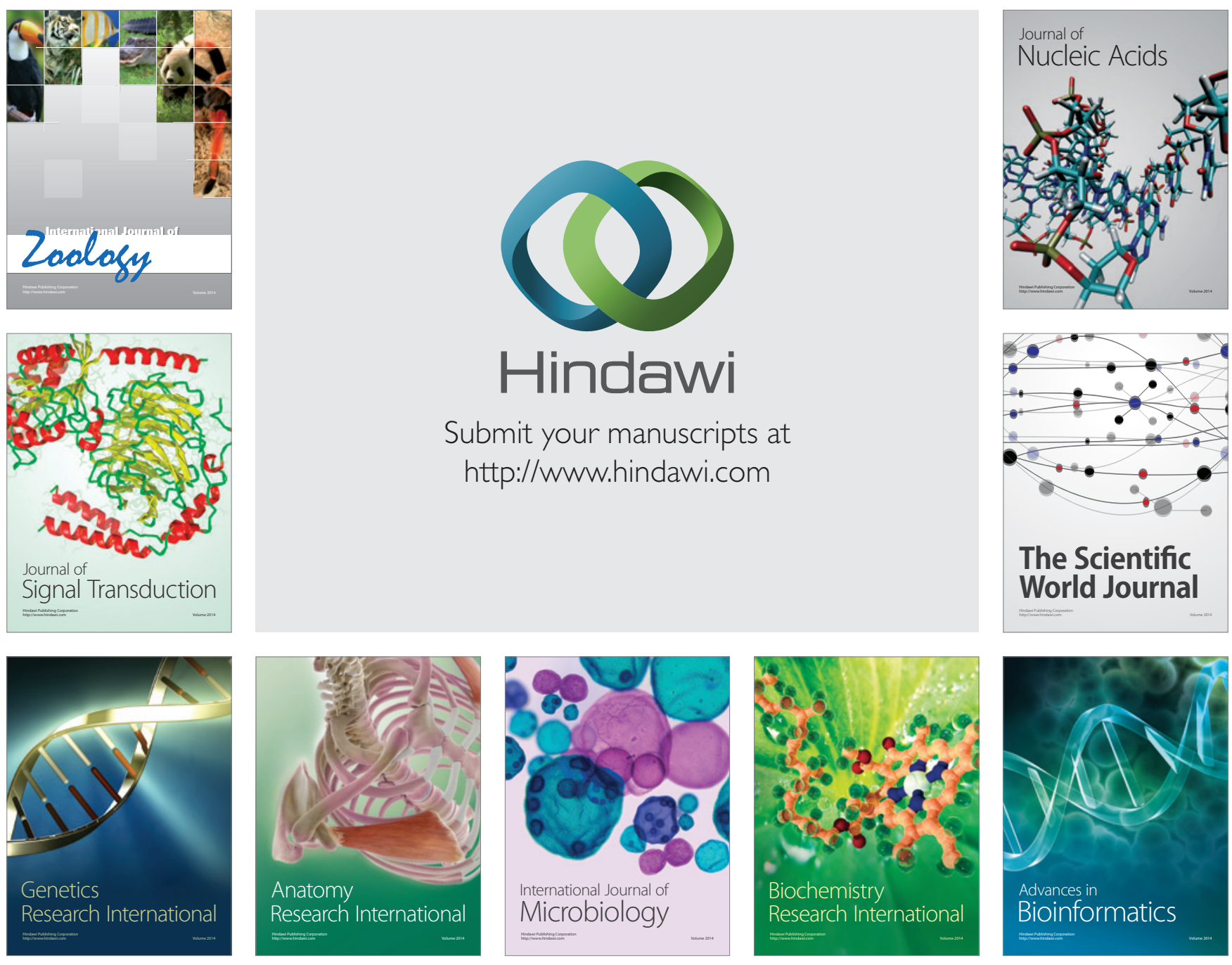

The Scientific World Journal
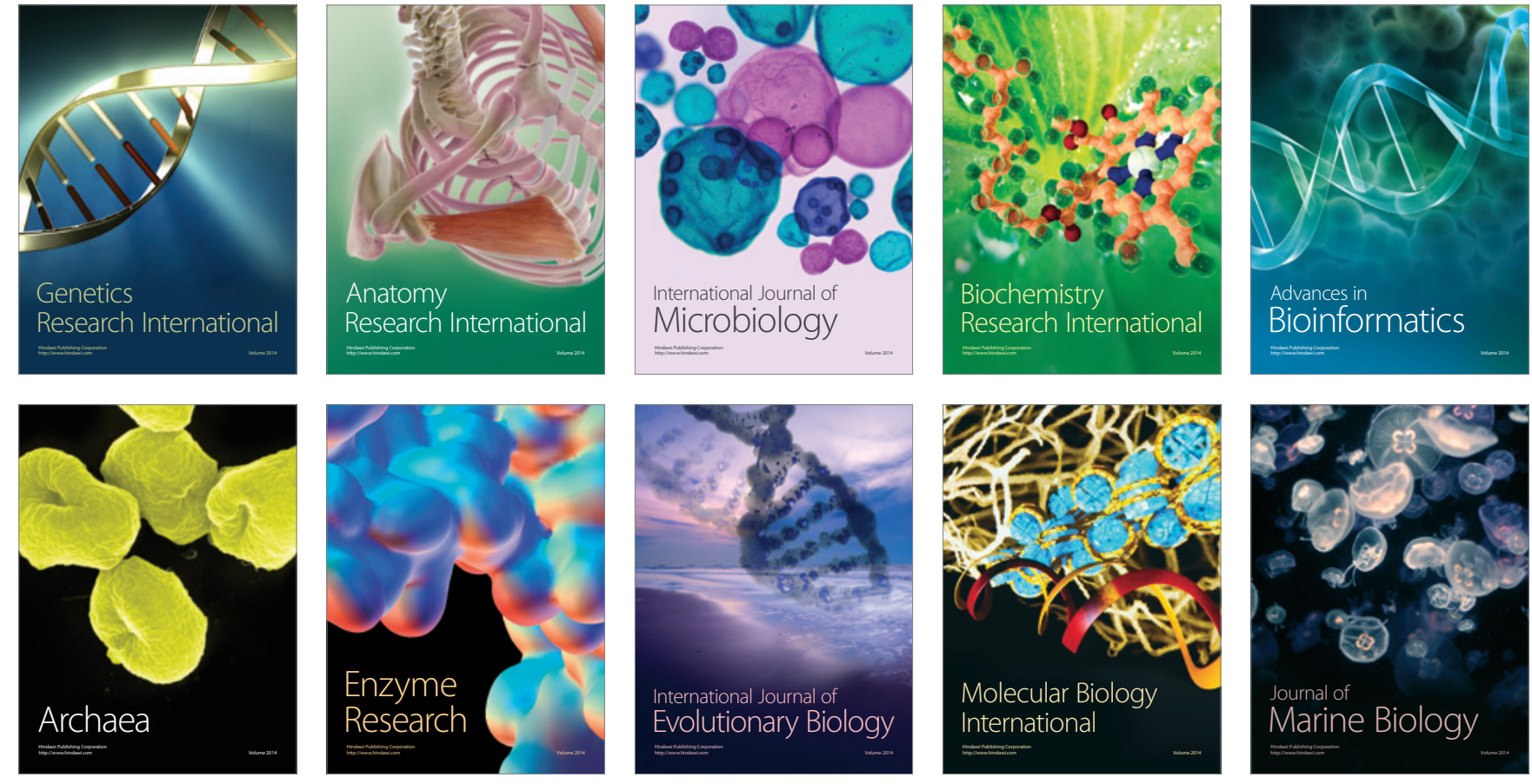